\title{
Prevalencia de Neuropatía Periférica en una Unidad de Diabetes
}

\author{
Prevalence of Peripheral Neuropathy in a Diabetes Unit \\ Prevalência de neuropatia periférica em uma unidade de diabetes
}

Roberto Di Lorenzi

ORCID: 0000-0003-1268-940X Internista. Diabetólogo. Ex asistente

Clínica Médica. ASSE.

Lorena Bruno ORCID: 0000-0001-5678-797 Internista. Diabetólogo. Ex asistente Clínica Médica. ASSE.

Maria Garau ORCID: 0000-0002-9632-7539

Profesora Agregada del

Departamento de Métodos

Cuantitativos. UdelaR.

Gerardo Javie ORCID: 0000-0002-5931-2055 Internista. Diabetólogo. ASSE.

Maria Eugenia Ruiz Diaz ORCID: 0000-0003-3297-2739 Internista. Diabetólogo. ASSE
Resumen: La principal complicación crónica de la diabetes mellitus es la neuropatía, en particular la polineuropatía diabética. El objetivo de este estudio fue evaluar la prevalencia de polineuropatía y su asociación a factores de riesgo y complicaciones crónicas en una unidad de diabetes. En una población de 81 pacientes diabéticos, se encontró una prevalencia de polineuropatía de $34.6 \%$. Es más frecuente y severa en la población de DM2, y se asoció con mayor tiempo de evolución de la enfermedad y complicaciones microangiopáticas. La polineuropatía sintomática predominó en el sexo femenino. Se concluye que la población diabética debe ser sometida a screening oportuno de polineuropatía.

Palabras claves: neuropatía diabética, neuropatía periférica, factores de riesgo, diabetes mellitus.

Abstract: The main chronic complication of diabetes mellitus is neuropathy, in particular diabetic polyneuropathy. The objective of this study was to evaluate the prevalence of polyneuropathy and its association with risk factors and chronic complications in a diabetes unit. In a population of 81 diabetic patients, a polyneuropathy prevalence of $34.6 \%$ was found. It is more frequent and severe in the T2DM population, and was associated with longer disease progression and microangiopathic complications. Symptomatic polyneuropathy predominated in the female sex. It is concluded that the diabetic population should undergo timely screening for polyneuropathy.

Key words: diabetic neuropathy, peripheral neuropathy, risk factors, diabetes mellitus.

Resumo: A principal complicação crônica do diabetes mellitus é a neuropatia, em particular a polineuropatia diabética. $O$ objetivo deste estudo foi avaliar a prevalência de polineuropatia e sua associação com fatores de risco e complicações crônicas em uma unidade de diabetes. Em uma população de 81 pacientes diabéticos, foi encontrada uma prevalência de polineuropatia de $34,6 \%$. É mais frequente e grave na população com DM2 e esteve associada a maior progressão da doença e complicações microangiopáticas. A polineuropatia sintomática predominou no sexo feminino. Conclui-se que a população diabética deve ser submetida a uma triagem oportuna da polineuropatia.

Palavras-chave: neuropatia diabética, neuropatia periférica, fatores de risco, diabetes mellitus.

Recibido: 09/08/2019 Aceptado: 23/05/2020

Unidad Docente Asistencial - Atención integral del paciente diabético. Hospital Pasteur. Administración Servicios del Estado (ASSE). Facultad de Medicina. Universidad de la República. UdelaR. Uruguay.

Correspondencia: E-mail: dilo@vera.com.uy 


\section{Introducción}

La diabetes se ha convertido en uno de los mayores problemas de salud mundial del siglo $X X I$. (1) $\mathrm{L}$ a prevalencia de diabetes en los países de América Latina se estima en un $8 \%$ de la población general, de los cuales aproximadamente un $50 \%$ no tiene diagnóstico. (2)

Una de las principales complicaciones crónicas es la neuropatía diabética, que se manifiesta con diversos patrones clínicos, siendo la polineuropatía diabética (PND) la forma más frecuente de presentación. ${ }^{(3)}$

La PND es uno de los factores responsables del pie diabético, determinante de discapacidad, de ulceraciones y amputaciones, responsable de alteraciones de la marcha y lesiones relacionadas con caídas. ${ }^{(4,5)}$ Es por tanto una situación clínica que incide directamente sobre la calidad y expectativa de vida de estos pacientes.

Por otro lado, pese a que puede estar presente incluso en prediabetes (glucemia de ayuno alterado, intolerancia a hidratos de carbono), suele ser la repercusión más tardíamente diagnosticada. ${ }^{(6)}$

Su prevalencia es difícil de establecer debido a la ausencia de criterios diagnósticos unificados, a la multiplicidad de métodos diagnósticos y a la heterogeneidad de las formas de presentación clínica. ${ }^{(7-9)}$

Se considera que del 50 al $75 \%$ de los diabéticos, padecerán neuropatía entre 5 a 10 años posteriores al inicio de la enfermedad, afectando tanto a pacientes con Diabetes Mellitus tipo 1 (DM1) como con Diabetes Mellitus tipo 2 (DM2).

Se estima que de cada 100 paciente con diabetes, 25 refieren síntomas de neuropatía, en 50 se demuestra afección neuropática en el examen físico y casi en 90 hay señales de afectación neuropática en las pruebas neurofisiológicas. ${ }^{(10)}$

La evolución y gravedad de la PND se correlaciona con el mal control metabólico de la enfermedad. Los factores de riesgo tradicionalmente relacionados son: hiperglucemia, aumento del valor de la hemoglobina glicosilada $(\mathrm{HbA} 1 \mathrm{C})$, edad del paciente y el tiempo de evolución de la diabetes. ${ }^{(10)}$

El factor modificable más fuertemente asociado a la aparición de PND es la hiperglucemia y su duración. Existen evidencias que el estricto control glucémico puede prevenir la aparición de la PND o enlentecer su evolución, por lo que debe considerarse el principal factor de riesgo.

Este enunciado está demostrado en el estudio DCCT en DM1, donde el tratamiento intensivo con insulina redujo la incidencia de neuropatía clínica en un $60 \% .{ }^{(11)}$

El estudio UKPDS en DM2 demostró que el tratamiento intensivo de la glucemia reducía el riesgo de complicaciones microvasculares en un $25 \%$, fundamentalmente retinopatía y nefropatía. ${ }^{(12,13)} \mathrm{A}$ la luz de estos resultados y de nuevos estudios se determinó que el tratamiento de la hiperglucemia en DM1 reduce la incidencia de neuropatía; sin embargo, en DM2 el control de la glucemia "per se" determina sólo una reducción marginal de 5 a $7 \%$ en el desarrollo de la misma.

Otros factores de riesgo modificables en el desarrollo de PND, tal como lo demostró el estudio EURODIAB son hipertensión arterial, dislipemia y obesidad. ${ }^{(13)}$

Factores de riesgo adicional son el tabaquismo, resistencia a la insulina ehipovitaminosis D. ${ }^{(14,15)}$

La evaluación de síntomas neuropáticos utilizando cuestionarios validados y pruebas clínicas, junto a una historia detallada para excluir otras etiologías, constituyen el pilar diagnóstico de la PND.

Se debe contar al menos con dos pruebas positivas, las mismas son la demostración de déficit sensitivos y/o motores a la percepción cutánea del calor/ frío, termoalgesia, presión, vibratoria, dolor punzante o alteración de los reflejos..$\left.^{(16-19}\right)$

Otros estudios de mayor sensibilidad diagnóstica pero que habitualmente no se usan en la práctica clínica son los estudios sensoriales cuantitativos, neurofisiológicos, biopsia cutánea y microscopia focal concorneal (MFC). ${ }^{(20,21)}$ 
Dada la alta prevalencia de la neuropatía diabética como complicación crónica, la relevancia de sus consecuencias, así como la ausencia de publicaciones en nuestro medio, se realiza el presente estudio en el ámbito de una unidad de diabetes.

Los objetivos son establecer la prevalencia de PND en la Unidad de Diabetes, relacionarlo con la enfermedad diabética del paciente, valorar la asociación de la misma con otras complicaciones crónicas y evaluar el rendimiento diagnóstico de NDS (Neuropathy Disability Score).

\section{Material y métodos}

Se realizó un estudio descriptivo, observacional, transversal, entre setiembre y noviembre de 2018 en la Unidad de diabetes de un hospital general.

Criterios de inclusión: todos los pacientes con DM1 y DM2, entre 15 y 70 años, que por lo menos hubieran consultado en dos oportunidades en el año anterior al estudio, que dieron consentimiento informado para el estudio.

Criterio de exclusión: antecedentes de amputación de miembros inferiores (MMII), enfermedad renal crónica conocida estadio $V$ (filtrado glomerular $<15 \mathrm{ml} / \mathrm{min}$ ) y /o paciente en hemodiálisis), alcoholismo (consumo etanol mayor 30gr/día en el hombre y $20 \mathrm{gr} /$ día en la mujer), arteriopatía de MMII (documentada por doppler arterial o informe cirujano vascular), hipotiroidismo sin tratamiento, enfermedades del tejido conectivo, enfermedades vertebro medulares (demostrada por estudio de imágenes TAC, RNM o estudio de conducción nerviosa), úlceras definidas oportunamente que impidan examen neurológico .

Las variables analizadas fueron: edad, sexo, tiempo de la diabetes, HbA1C, tabaquismo, obesidad (IMC), dislipemia y repercusiones crónicas.

Tabaquismo: adicción crónica generada por el tabaquismo que produce dependencia física y psicológica así como gran número de enfermedades. Se considera fumador hasta 6 meses de abandonar el hábito.

Obesidad valorada de acuerdo a IMC: bajo peso < $18.49 \mathrm{~kg} / \mathrm{m} 2$; normopeso: 18.5 - $24.9 \mathrm{~kg} /$ m2; sobrepeso 25 - 29.9 kg/m2; obesidad > $30 \mathrm{~kg} / \mathrm{m} 2$.

Retinopatía: informe oftalmológico.

Dislipemia: LDL > 100 mg/dl; triglicéridos > 150mg/dl .

Enfermedad renal crónica (ERC): creatinina $>1,5 \mathrm{mg} / \mathrm{dl}$ o proteinuria positiva o $\mathrm{FG}<60 \mathrm{ml} /$ $\min$.

Los test utilizados para el diagnóstico de PND, como la escala de síntomas TSS (total Sympton Score) y de signos NDS (Neuropathy Disability Score), son validados a nivel internacional, utilizándose en distintos ensayos clínicos.

El diagnóstico de PND se estableció de la siguiente manera:

1) NDS $\geq 6$, sin importar el puntaje de TSS

2) NDS de 3 a 5 con un puntaje de TSS $\geq 5$

NDS: Es una escala de 4 signos: percepción de temperatura, vibración, presión y reflejo aquilino. Se realizó una modificación, utilizada en otro estudio, que consistió en evaluar la presión a través de un monofilamento de $10 \mathrm{gr}$. El test original usa el pinchazo con alfiler que actualmente está en desuso ya que puede causar lesiones en los pacientes con sensibilidad disminuida. En cambio el monofilamento no tiene este inconveniente, está validado como herramienta y su uso es universal. El puntaje para cada signo es: 0 si la percepción está conservada, 1 si está alterada, con excepción del reflejo Aquileo, que es 0 si es normal, 1 si está disminuido y 2 si está ausente (Anexo 1).

TSS: Es una escala que contempla 4 síntomas: dolor quemante, dolor lancinante, adormecimiento y hormigueo o parestesias. Se les adjudica un puntaje, teniendo en cuenta la frecuencia y severidad de cada uno (22) (Anexo2).

La recolección de los datos, así como la evaluación de síntomas y signos fue realizada por diabetólogos entrenados. 
Las variables cualitativas se presentan como valores absolutos y porcentajes, las variables cuantitativas se resumen con la media \pm desvío estándar (DS). Para investigar asociación entre variables cualitativas se utilizó el test de chi cuadrado y para comparar variables cuantitativas el test de Mann Whitney. Valores $p<5 \%$ se consideraron significativos.

\section{Resultados}

Se analizaron 81 pacientes que cumplían los criterios de inclusión. En la tabla 1 se presentan las características epidemiológicas de los mismos.

\begin{tabular}{ll|l|}
\hline & DM 1(n=18) & DM 2 ( $\mathbf{n = 6 3 )}$ \\
\hline Sexo femenino & $11(61,1 \%)$ & $34(53,9 \%)$ \\
\hline $\begin{array}{l}\text { Edad (años) } \\
\text { Mediana (rango) }\end{array}$ & $\begin{array}{l}57(35-70) \\
56,3 \pm 7,9\end{array}$ \\
$\quad \begin{array}{l}\text { Media } \pm \text { DS } \\
\text { Tiempo evolución DM (años) }\end{array}$ & \\
$\quad 32,5 \pm 16,0$ & $5(1-28)$ \\
Mediana (rango) & $8(1-41)$ & $7,1 \pm 7,1$ \\
\hline
\end{tabular}

En la tabla 2 se presentan los hallazgos del control metabólico determinado por el valor de la $\mathrm{HbA1C}$.

\begin{tabular}{|c|c|c|}
\hline & DM $1(n=18)$ & DM $2(n=63)$ \\
\hline \multicolumn{3}{|l|}{ HbA1C (\%) } \\
\hline Mediana (rango) & $8,0(6,8-10,9)$ & $7,7(5,2-14,0)$ \\
\hline Media \pm DS & $8,5 \pm 1,3$ & $8,0 \pm 1,9$ \\
\hline
\end{tabular}

En la tabla 3 se muestra la presencia de otros factores de riesgo modificables

\begin{tabular}{ll|l|}
\hline & DM $\mathbf{1}(\mathbf{n}=\mathbf{1 8})$ & DM $\mathbf{2}(\mathbf{n}=\mathbf{6 3})$ \\
\hline Tabaquismo & $3(16,7 \%)$ & $15(25,0 \%)$ \\
\hline $\begin{array}{l}\text { IMC (kg/m2) } \\
\text { Mediana (rango) }\end{array}$ & $\begin{array}{l}31,4(22-55) \\
33,7 \pm 7,8\end{array}$ \\
Media \pm DS & $24,0(19-34)$ & \\
\hline $\begin{array}{l}\text { Grupo IMC } \\
\text { Normopeso }\end{array}$ & $25,0 \pm 4,1$ & $5(9,1 \%)$ \\
Sobrepeso & $11(20,2 \%)$ \\
Obesidad & $10(58,8 \%)$ & $39(70,9 \%)$ \\
\hline Dislipemia & $5(27,8 \%)$ & $45(71.4 \%)$ \\
\hline
\end{tabular}

Se encontró que 4/18 (22,2\%) de los pacientes DM1 y 7/63 (11,1\%) de los pacientes DM2 presentaban retinopatía diabética, mientras que $2 / 18(11,1 \%)$ de los DM1 y 10/63 $(15,9 \%)$ de los DM2 tenían ERC.

La presencia de PND estaba presente en 28/81 pacientes (34,6\%).

En la tabla 4 se presentan las características epidemiológicas de esta población.

\begin{tabular}{|c|c|c|c|}
\hline & Con PND $\mathrm{n}=\mathbf{2 8}$ & $\operatorname{Sin}$ PND $\mathrm{n}=53$ & Valor $\mathrm{p}$ \\
\hline Sexo femenino & $14(50 \%)$ & $31(58,4 \%)$ & $0.46 \dagger$ \\
\hline $\begin{array}{l}\text { Edad (años) } \\
\text { Mediana (rango) } \\
\text { Media } \pm \text { DS }\end{array}$ & $\begin{array}{l}56,5(34-71) \\
56,0 \pm 9,4\end{array}$ & $\begin{array}{l}54,0(15-70) \\
48,4 \pm 15,8\end{array}$ & 0,0569 \\
\hline Diabetes 2 & 23 & 40 & $0,49 \dagger$ \\
\hline $\begin{array}{l}\text { Tiempo evolución DM (años) } \\
\text { Mediana (rango) } \\
\text { Media } \pm \text { DS }\end{array}$ & $\begin{array}{l}8,5(1-40) \\
13,4 \pm 11,5\end{array}$ & $\begin{array}{l}4(1-41) \\
6,0 \pm 6,7\end{array}$ & 0,0029 * \\
\hline
\end{tabular}

† Test de chi cuadrado ๆ Test U de Mann Whitney * Valor $p$ significativo 
En el gráfico 1 se presenta la relación entra la presencia de PND y la edad del paciente.

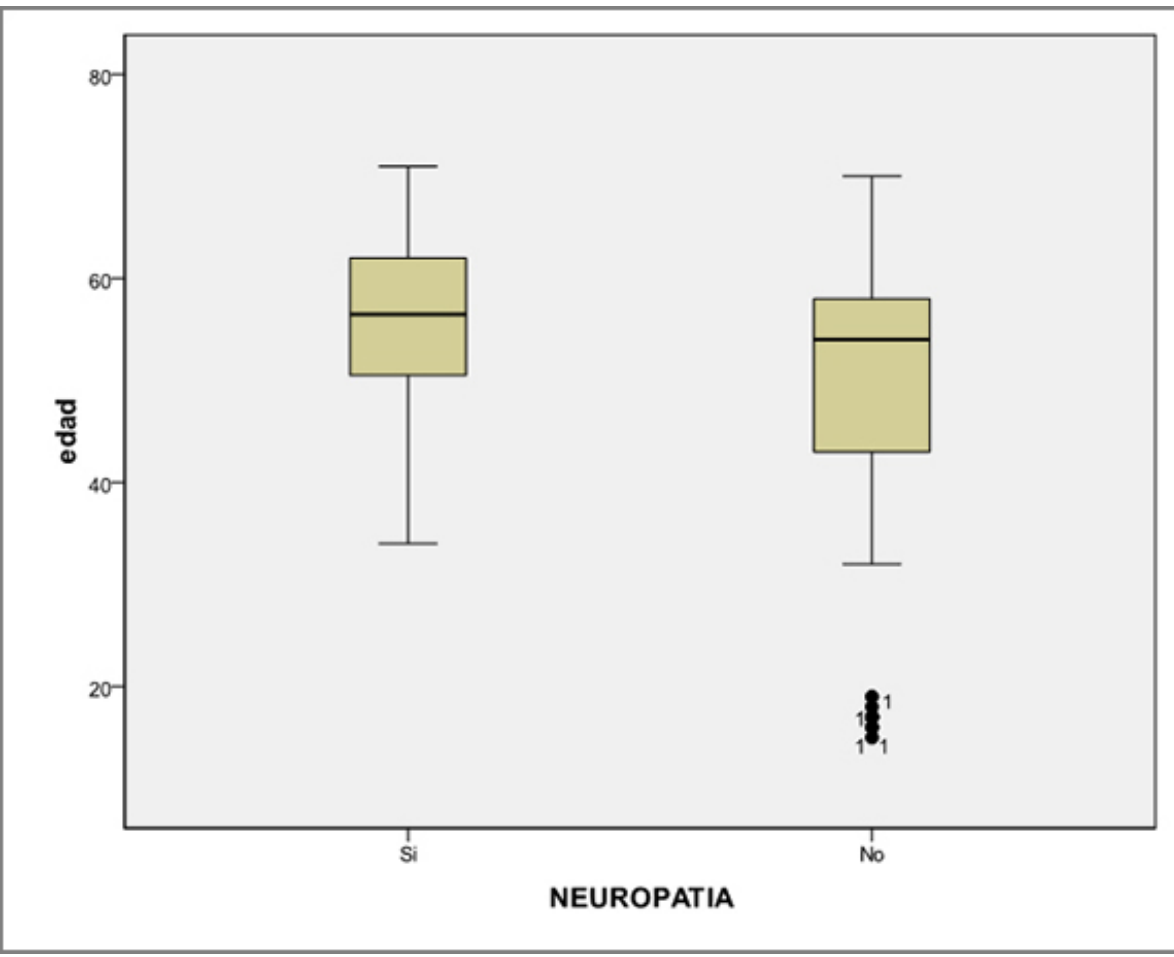
DM.

En el gráfico 2 se representa la presencia de PND en relación al tiempo de evolución de la

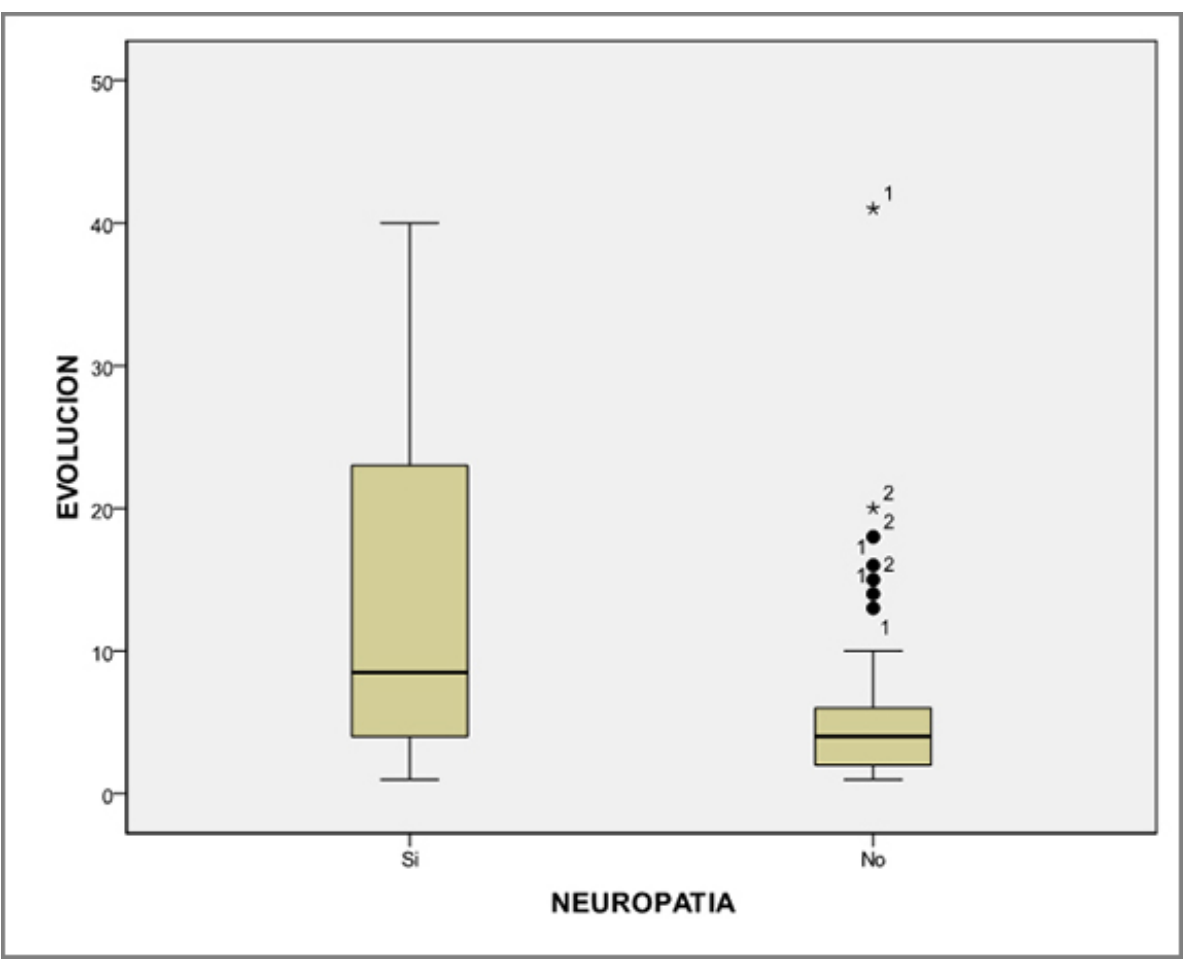

En la tabla 5 se presentan los hallazgos del control metabólico determinado por el valor de la $\mathrm{HbA1C}$. 


\begin{tabular}{|c|c|c|c|c|}
\hline & & Con PND n=28 & $\operatorname{Sin}$ PND $n=53$ & Valor p \\
\hline $\begin{array}{l}\text { Tabla 5: Control metabólico de la } \\
\text { población con y sin PND. }\end{array}$ & $\begin{array}{l}\text { HbA1C (\%) } \\
\quad \text { Mediana (rango) } \\
\text { Media } \pm \text { DS }\end{array}$ & $\begin{array}{l}8,4(5,7-12,0) \\
8,4 \pm 1,7\end{array}$ & $\begin{array}{l}7,6(5,2-14,0) \\
8,0 \pm 1,9\end{array}$ & 0,189 \\
\hline
\end{tabular}

† Test de chi cuadrado ๆ Test $U$ de Mann Whitney * Valor $p$ significativo

En el gráfico 3 se representa la relación entre la presencia de PND y el control metabólico según $\mathrm{HbA1C}$.

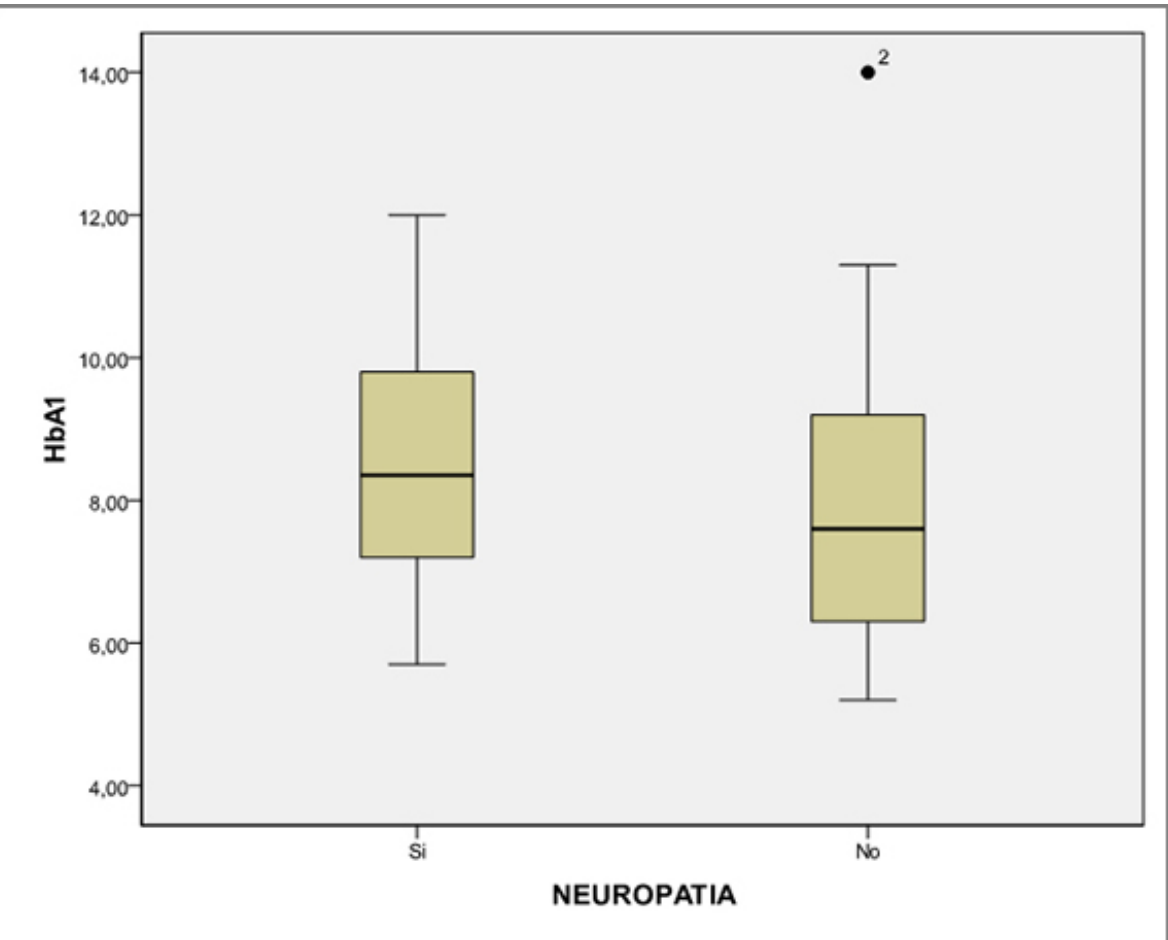

En la tabla 6 se muestra la presencia de otros factores de riesgo modificables.

\begin{tabular}{ll|l|l|}
\hline & Con PND $n=28$ & Sin PND $n=53$ & Valor $\mathbf{p}$ \\
\hline Tabaquismo & $4(14,3 \%)$ & $14(26,4 \%)$ & $0,21 \dagger$ \\
\hline Grupo IMC & & & $0,43 \dagger$ \\
$\quad$ Normopeso & $5(21,7 \%)$ & $10(20,4 \%)$ & \\
Sobrepeso & $3(13,0 \%)$ & $13(26,5 \%)$ & \\
Obesidad & $15(65,2 \%)$ & $26(53,1 \%)$ & \\
\hline Dislipemia & $15(53,6 \%)$ & $32(60,4 \%)$ & $0,89 \dagger$ \\
\hline
\end{tabular}

† Test de chi cuadrado ๆ Test U de Mann Whitney * Valor $\mathrm{p}$ significativo

En la tabla 7 se muestra la presencia de otras repercusiones de estos pacientes.

Tabla 7: Repercusiones de la población.

\begin{tabular}{l|l|l|l|}
\hline & Con PND $n=28$ & Sin PND $\mathbf{n = 5 3}$ & Valor p \\
\hline ERC & $7(25,0 \%)$ & $5(9,4 \%)$ & $0,06 \dagger$ \\
\hline Retinopatia & $8(28,6 \%)$ & $3(5,7 \%)$ & $0,005 \dagger^{*}$ \\
\hline
\end{tabular}

† Test de chi cuadrado ๆ Test $U$ de Mann Whitney * Valor $p$ significativo

El diagnóstico se realizó en 27 casos (96.5\%) por el criterio de la escala NDS $\geq 6$, y solo en 1 caso fue por la escala TSS $\geq 5$ (NDS 3-5).

Al evaluar el rendimiento diagnóstico de los signos considerados en el NDS en el grupo de pacientes con PND, se encontró que el 100\% tenía alteración de los reflejos osteotendinosos, el $89 \%$ presentó apalestesia, el $44 \%$ tenía ausencia de respuesta al monofilamento y el $17.9 \%$ tenía pérdida de la termosensibilidad. 
Del total de pacientes con PND, 18/28 (64\%) presentaba síntomas. Esta subpoblación sintomática incluyó a 15/18 pacientes con DM2 (83\%) y 3/18 con DM1 (17\%); corresponden al sexo femenino 10/18 (55,6\%). No se encontró asociación significativa entre sexo y síntomas (valor $\mathrm{p}=0.7$ en DM1 y 0,07 en DM2).

Un número no despreciable de pacientes 17/53 (32\%) presentaba alteraciones en el test NDS (valor 3-5); es decir que presentaban alteraciones al examen, pero no alcanzan el diagnóstico de neuropatía por los criterios establecidos.

\section{Discusión}

La población analizada en este estudio es mayoritariamente de sexo femenino, y tal cual se conoce de la epidemiología de esta enfermedad, predomina la DM2, con edades y tiempo de evolución acordes al tipo de diabetes analizada.

Tanto en los pacientes con DM1 como DM2 se constata un mal control metabólico con valores de $\mathrm{HbA} 1 \mathrm{C}$ por encima de los objetivos.

La presencia de sobrepeso/obesidad y dislipemia presentan alta prevalencia en DM2 (91.1\% y $71,4 \%$ respectivamente), elementos que apuntan al alto riesgo cardiovascular de esta población. Se destaca la presencia de sobrepeso /obesidad y dislipemia en DM1 que llega al $38,9 \%$ y $11 \%$ respectivamente, lo cual determina un llamado de atención y nos lleva a tener un enfoque proactivo en la corrección de estos factores también en pacientes con DM1.

El porcentaje de pacientes fumadores con neuropatía es más bajo que el de aquellos sin neuropatía, no comportándose con un factor de riesgo jerárquico en nuestra población de pacientes con PND.

La prevalencia de PND en nuestra serie fue de $34.6 \%$, cifras superiores al $25 \%$ encontrado por Mendivil en Colombia en población con DM2(23) y al 22\% del trabajo de Sarturi-Tres en Brasil. (24) Sin embargo otros estudios como el de Aliss Samur e Ibarrar enMéxico encontraron una prevalencia de PND mayor $81 \%$ y $69 \%$ respectivamente. ${ }^{(25,26)}$

Esta variabilidad la podemos explicar por los pacientes incluidos en los distintos estudios y los criterios diagnósticos empleados en cada uno de ellos.

No encontramos relación estadísticamente significativa cuando se analiza el sexo. Este hallazgo tampoco ha sido encontrado en otros reportes. ${ }^{(27)}$

Cuando analizamos aquellos factores que la literatura relaciona con la presencia de PND, como son la edad, control metabólico y tiempo de evolución de la diabetes, en nuestro estudio no se comprueba asociación estadística con respecto a la edad de los pacientes ni con el control metabólico, si bien el grupo de pacientes con PND presentaba una media de HbA1C superior al grupo sin PND (8,4\% vs $8.0 \%)$.

Uno de los elementos que pudo haber incidido en nuestros resultados es que consideramos en forma aislada la última determinación de $\mathrm{HbA} 1 \mathrm{C}$ y no el promedio de los últimos años, tal cual fue considerado en otros estudios. ${ }^{(21)}$

Los trabajos actuales indican que un mejor control metabólico, es decir la reducción de la $\mathrm{HbA1c}$, se asocia con disminución en la prevalencia de PND en población con DM1, pero no en DM2, donde son múltiples los factores involucrados en el desarrollo de PND. (10-12)

Sin embargo el análisis del tiempo de evolución de la diabetes sí se asoció significativamente con el diagnóstico de PND (p0,002), tanto en DM1 como en DM2, confirmando que es un factor de riesgo importante y bien reconocido. ${ }^{(15)}$

De los otros factores relacionados con la presencia de PND, no se encontró asociación significativa en el análisis del tabaquismo, sobrepeso/obesidad y dislipemia. Los resultados de otros trabajos son también no concluyentes con respecto a éstos factores. ${ }^{(28-30)}$

El análisis de otras repercusiones microangiopáticas, evidenció una asociación significativa de PND con retinopatía (p0,005), pero no con ERC - aunque ésta fue más frecuente en el grupo con PND $(25.0 \%$ vs $9.4 \%)$.

En relación a la metodología, encontramos un alto rendimiento diagnóstico de los instrumentos utilizados para evaluar reflejos y sensibilidad vibratoria (100\% y $89 \%$ respectivamente); $\sin$ 
embargo, este rendimiento no llega al 50\% cuando hablamos de la sensibilidad a la presión (utilizando el monofilamento) y la sensibilidad térmica.

Particular mención merece el uso del monofilamento ampliamente utilizado como screening de neuropatía y utilizado en distintos trabajos clínicos. Un metaanálisis reciente encuentra una sensibilidad limitada para la detección de PND, concluyendo que el uso clínico del monofilamento no se puede alentar basándose en la evidencia actualmente disponible. ${ }^{(31)}$

Visto que la tercera parte de los pacientes sin PND presentaba de todas maneras alteraciones en algunos de los signos del NDS, nos lleva a plantear que si contáramos con métodos de mayor rendimiento diagnóstico, como los estudios cuantitativos sensoriales, biopsia de piel o MFC, optimizaríamos el diagnóstico, siendo mayor la prevalencia encontrada de PND. ${ }^{(19)}$

La PND sintomática se encontró más frecuentemente en DM2 y en el sexo femenino; si bien este hallazgo no fue significativo, está en concordancia con la bibliografía. Esto podría explicarse por diferencias en los umbrales al dolor; también debemos tener presente que se trata de manifestaciones subjetivas del individuo, necesariamente relacionadas con experiencias vividas anteriormente. Los trabajos muestran predominio en las mujeres, proponiéndose diferentes explicaciones. ${ }^{(30-40)}$

Consideramos que se trata de un trabajo que muestra las características de nuestra población, que tiene por fortaleza estrictos criterios de exclusión lo que por un lado reduce la posibilidad de factores de confusión en el diagnóstico de PND, pero por otro, limita el número de pacientes incluidos en el trabajo, ya que este grupo de pacientes es seguramente el que presenta mayor daño neuropático.

Otro elemento que consideramos de importancia ha sido el utilizar para el diagnóstico de PND test validados internacionalmente con criterios clínicos. Apostamos a contar en el futuro con otros test diagnósticos, que permitan optimizar el diagnóstico de esta enfermedad.

\section{Conclusiones}

La Prevalencia de PND fue del 34.6\%. Se encontró asociación significativa entre la presencia de PND y con el tiempo de evolución de la DM. Es necesario el screening oportuno de PND con instrumentos validados dado que un porcentaje no despreciable de los casos diagnosticados (36\%) no presentaba síntomas.

\section{Bibliografía}

1- Juster-Switlyk K, Smith GA. Updates in diabetic peripheral neuropathy. F1000Research. 2016. 5 (F1000 Faculty Rev): 738. 25. doi:10.12688/f1000research.7898.1

2- Brasil. Ministreio da Saude .Secretaria de Atenco a Saude .Departamento de Atencao Basica. Diabetes Mellitus.(Serie A. Normas e Manuals Tecnicos). Cuadernos de Atencao Basico . MSP: Brasilia; 2006. p. $16: 56$

3- Pop-Busui R, Boulton AJM, Feldman EL, Bril V, Freeman R, Malik RA, et al .Diabetic Neuropathy:A Position Statment by the Americam Diabetes Association. Diabetes Care. 2017 Jan;40(1):136-154. doi: $10.2337 / \mathrm{dc} 16-2042$.

4- Alam U, Riley DR, Jugdey RS, Azmi S, Rajbhandari S, D'Août K, et al. Diabetic Neuropathy and Gait: A Review. Diabetes Ther. 2017 Dec; 8(6): 1253-1264.

5- Sanz Garbalan I, Lazaro-Martinez JL, Garcia-Morales E, Molines-Barroso R, Alvaro-Afonso F, GarciaAlvarez Y. Advantages of early diagnosis of diabetic neuropathy in the prevention of diabetic foot ulcers. Diabetes Res Clin Pract. 2018 Dec; 146:148-154. doi: 10.1016/j.diabres.2017.12.018.

6- Stino, AM, Smith AG. Peripheral neuropathy in prediabetes and the metabolic syndrome. J Diabetes Investig. 2017 Sep;8(5):646-655. doi: 10.1111/jdi.12650

7- de Brito Gomes M, Gianella D, Faria M, Tambascia M, Fonseca RM, Réa R, et al. Prevalence of type 2 diabetic patients within the targets of care guidelines in daily practice: a multicenter study in Brazil. Rev Diabet Stud. 2006 Summer; 3(2): 82-87.

8- Boulton AJM, Vinik Al, Arezzo JC, Feldman EL, Freeman R, Malik RD, et al. Diabetic Neuropathies .A statement by the American Diabetes Association Diabetes Care. 2015; 28: 956-62.

9- Escaño-Polanco FM, Odriozola A, Davidson J, Pedrosa H, Fuente G, Márquez G, et al. Consenso de expertos para el manejo de la neuropatía diabética. Rev ALAD. 2016; 6:121-50 
10- Tesfaye S, Chaturvedi N, Eaton SE, Ward JD, Manes C, lonescu-Tirgoviste C, et al. Vascular risk factors and diabetic neuropathy. N Engl J Med. 2005; 352(4):341-50. This study demonstrates potential risk factors of DPN in a cohort of 3250 T1DM participants. [PubMed: 15673800]

11- Grisold A, Callaghan BC and Feldman EL. Mediators of Diabetic neuropathy is hyperglycemia The Only Culprit? Curr Opin Endocrinol Diabetes Obes .2017;24(2):103-111.

12- Bianchi C, Del Prato S. Metabolic Memory and Individual Treatment Aims in Type 2 Diabetes OutcomeLessons Learned from Large Clinical Trials. Rev Diabet Stud. 2011; 8(3):432-440.

13- Pop-Busui R, Herman WH, Feldman EL, Low PA, Martin CL, Cleary PA, et al .DCCT and EDIC Studies in Type 1 Diabetes: Lessons for Diabetic Neuropathy Regarding Metabolic Memory and Natural History. Curr Diab Rep. 2010Aug; 10(4):276-282.

14- Perez Matos MC, Morales MC, Mendivil CO. Review Article Lipids: A Suitable Therapeutic Target in Diabetic Neuropathy? J Diabetes Res. 2017; 2017: 6943851.

15- Papanas N, Ziegler D. Risk Factors and comorbidities in Diabetic Neuropathy; An Update 2015. Rev Diabet Stud. Spring-Summer 2015;12(1-2):48-62. doi: 10.1900/RDS.2015.12.48.

16- Shillo P, Sloan G, Greig M, Hunt L, Selvarajah D, et al. Painful and Painless Diabetic Neuropathies. What is the Difference? Curr Diab Rep. 2019 May 7;19(6):32. doi: 10.1007/s11892-019-1150-5.

17- Boulton AJM, Armstrong DG, Albert SF, Frykberg RG, Hellman R, Kirkman MS, et al. Comprehensive Foot Examination and Risk Assessment: A Report of the Task Force of the Foot Care Interest Group of the American Diabetes Association, With Endorsement by the American Association of Clinical Endocrinologists. Diabetes Care. 2008; 31 (suppl 1):S1679-S85.

18- Thomas PK. Classification, differential diagnosis and staging of diabetic peripheral neuropathy. Diabetes .1997; 46(suppl 2): S54-S57

19- Chikai M, Ozawa E, Takahashi N, Nunokawa K. Evaluation of the variation in sensory test results using Semmes-Weinstein monofilaments. Med Biol Soc. 2015 August; 2015:1259-62

20- Azmi S, Petropoulos IN, Ferdousi M, Ponirakis G, Alam U, Malik RA. An update on the diagnosis and treatment of diabetic somatic and autonomic neuropathy. F1000Res. 2019; 8: F1000 Faculty Rev-186. doi:10.12688/f1000 research.17118.1.

21- Petropoulus IN, Ponirakis G, Malik RA. Diagnosing Diabetic Neuropathy: Something Old; Something New. Diabetes Metab J 2018;42:255-269.

22- Meijer JWE, Smit AJ, Sonderen EV, Groothoff JW, Eisma WH, Links TP. Symptom scoring systems to diagnose distal polyneuropathy in diabetes: the Diabetic Neuropathy Symptom score. Diabet Med. 2002 Nov;19(11):962-5.

23- Mendivil CO, Viscaina E, Hernandez JD, Rodriguez M, Franco H. Polineuropatía diabética : Prevalencia y factores asociados en una población colombiana. Rev Alad. 2013; 2(2).

24- Sarturi Tres G, Kurtz Lisbôal HR, Syllosll R, Cananill LH, Gross JL. Prevalence and characteristics of diabetic polyneuropathy in Passp Fundo, South of Brasil. Arq Bras Endocrinol Metab. 2007; 51: 987992.

25- Aliss Samur JA, Cervantes MZ, Ibarra A, Gonzalez Barcena D. Prevalencia de neuropatía periférica en diabetes mellitus. Acta Medica Grupo Angeles.2006; 4(1)

26- Ibarrar C, De Jesus J, Hernandez R, Nieves R, Leiva R. Prevalencia de neuropatía periférica en diabéticos tipo 2 en el primer nivel de atencion. Rev Med Chile. 2012; 140:1126-1131.

27- Ugoya SO. Clinically diagnosed diabetic neuropathy: frecuency, types and severity. J Natl Med Assoc. 2006;98(11):1763-61.

28- Ziegler D. Prevalence of polyneuropathy in pre-diabetes and diabetes is associated with abdominal obesity and macroangiopathy: the MONICA /KORA Augsburg Surveys S2 and S3. Diabetes Care .2008;31(3):464-9.

29- Callaghan BC, Cia R, Reynols E, Banerjee M, Rothberg AE, et al. Association Betwen Metabolic Syndrome Components and polyneuropathy in an Obese Population. Jama Neurol 2016.

30- Callaghan BC, Gao LL, Li Y, Zhou X, Reynolds E, Banerjee M, Pop-Busui R, Feldman EL, Ji L. Diabetes and obesity are the main metabolic drives of peripheral neuropathy. Ann Clin Transl Neurol. 2018; 5:397. 
Rev. urug. med. interna.

31- Wang F, Zhang J, Yu J, Liu S, Zhang R, Ma X, et al .Diagnostic Accuracy of monofilament Test for Detecting Diabetic Peripheral Neuropathy: A Systematic Review and Meta-Analisis. J Diabetes Res. 2017; 2017:8787261. doi: 10.1155/2017/8787261.

32- Shillo P, Sloan G, Greig M, Hunt L, Selvarajah D, Elliott J, et al.Painful and Painless Diabetic Neuropathies: What Is the Difference? Curr Diab Rep.2019;19(6):32.

33- Rey R. Tratamiento del Dolor neuropático: revisión de las últimas guías y recomendaciones. Neu Arg.2013;5(S1):S1-S7.

34- Dominguez C, Flores C, Fuentes G, Garcia C, Gimenes R, Houssay, Solange, et al. Actualización del Tratamiento de la Polineuropatía Diabética Periférica Dolorosa 2015.Comite de Neuropatía Diabética. Rev. Soc. Argent. Diabetes. 2016; 50(1): 35-46.

35- Hébert HL, Veluchamy A, Torrance N, Smith BH. Risk factors for neuropathic pain in diabetes mellitus. Pain. 2017; 158: 560-568.

36- Elliott J. Female sex: an independent risk factor for the development of painful neuropathy? Diabetes. 2006; 55(Supp 1).

37- Themistocleous AC, Ramirez JD, Shillo PR, Lees JG, Selvarajah D, Orengo C, et al. The pain in neuropathy study (PiNS): a cross-sectional observational study determining the somatosensory phenotype of painful and painless diabetic neuropathy. Pain. 2016; 157:1132-1145

38- Algeffari MA. Painful diabetic peripheral neuropathy among Saudi diabetic patients is common but under-recognized: multicenter cross-sectional study at primary health care setting. J Family Community Med. 2018; 25:43-47.

39- Ziegler D, Landgraf R, Lobmann R, Reiners K, Rett K, Schnell O, et al. Painful and painless neuropathies are distinct and largely undiagnosed entities in subjects participating in an educational initiative (PROTECT study) Diabetes Res Clin Pract. 2018; 139:147-154.

40- Pai YW, Lin CH, Lee IT, Chang MH. Prevalence and biochemical risk factors of diabetic peripheral neuropathy with or without neuropathic pain in Taiwanese adults with type 2 diabetes mellitus. Diabetol Metab Syndr. 2018; 12:11-16.

\section{Aporte cada autor al trabajo}

Roberto Di Lorenzi: Concepción y diseño del trabajo, recolección de datos, análisis e interpretación de los datos, redacción del manuscrito.

Lorena Bruno: Recolección de datos, revisión crítica del manuscrito

Maria Garau: Análisis estadístico de los datos, revisión crítica del manuscrito

Gerardo Javiel: Recolección de datos, revisión crítica del manuscrito.

Maria Eugenia Ruiz Diaz: Recoleccion de datos, revisión crítica del manuscrito 


\section{Anexos}

\section{Anexo 1: Neuropathy Disability Score (NDS modificado)}

\begin{tabular}{|l|l|}
\hline Puntuación máxima 10 puntos (ambos pies) \\
\hline Signos ligeros & $3-5$ puntos \\
\hline Signos moderados & $6-8$ puntos \\
\hline Signos severos & $9-10$ puntos \\
\hline
\end{tabular}

\begin{tabular}{l|l|l|l|l|l|l|}
\multirow{2}{*}{ NDS } & \multicolumn{4}{|l|}{ PD } & PI & \\
\hline \multirow{2}{*}{ Reflejo Aquileo } & normal & refuerzo & ausente & normal & refuerzo & Ausente \\
\cline { 2 - 7 } & 0 & 1 & 2 & 0 & 1 & 2 \\
\hline \multirow{2}{*}{ Monofilamento } & Normal & Ausente & normal & Ausente & \\
\cline { 2 - 7 } & 0 & 1 & 0 & 1 \\
\hline \multirow{2}{*}{ Vibración } & Normal & Ausente & normal & Ausente \\
\hline \multirow{2}{*}{ Temperatura } & 0 & 1 & 0 & 1 \\
\hline Total por pie & Normal & Ausente & normal & Ausente \\
\hline
\end{tabular}

\section{Anexo 2: Puntuación de todos los síntomas (Total Symptom Score)}

\begin{tabular}{|l|l|}
\hline Rasgos del TSS & \\
\hline 1.Dolor & Tiene dolor punzante o como descarga eléctrica \\
\hline 2.Ardor & Tiene dolor quemante en las piernas \\
\hline 3.Parestesias & Sensación de hormigueo o cosquilleo en los pies \\
\hline 4.Adormecimiento & Tiene entumecimiento o pérdida de sensibilidad \\
\hline
\end{tabular}

\begin{tabular}{|l|l|l|l|l|}
\hline \multicolumn{5}{|l|}{ Intensidad de sintomas (severidad) } \\
\hline Frecuencia de sintomas & Ausente & Leve & Moderado & Severo \\
\hline $\begin{array}{l}\text { Ocasional } \\
\text { (< a } \mathbf{1 / 3} \text { del tiempo }\end{array}$ & 0 & 1,00 & 2,00 & 3,00 \\
\hline $\begin{array}{l}\text { Frecuente } \\
\mathbf{1} / \mathbf{3} \text { a } \mathbf{2} / \mathbf{3} \text { del tiempo }\end{array}$ & 0 & 1,33 & 2,33 & 3,33 \\
\hline Continuo & 0 & 1,66 & 2,66 & 3,66 \\
\hline & $\begin{array}{l}\text { No hay } \\
\text { síntoma }\end{array}$ & $\begin{array}{l}\text { Síntoma } \\
\text { perceptible, } \\
\text { no interfiere } \\
\text { actividad diaria }\end{array}$ & $\begin{array}{l}\text { Síntoma interfiere } \\
\text { con actividad } \\
\text { diaria 0 con el } \\
\text { sueño, requiere } \\
\text { tratamiento }\end{array}$ & $\begin{array}{l}\text { El síntoma interfiere } \\
\text { frecuentemente con la } \\
\text { actividad diaria o con el sueño, } \\
\text { incapacitante o requiere } \\
\text { tratamiento para control }\end{array}$ \\
\hline TOTAL & & & & \\
\hline
\end{tabular}

